\title{
Des cheffes orchestrent le travail. Un regard sociomusicologique
}

Entretien avec Hyacinthe Ravet

Myrtille Picaud et Mathilde Provansal

\section{CpenEdition}

Journals

Édition électronique

URL : http://journals.openedition.org/transposition/1241

DOI : 10.4000/transposition. 1241

ISSN : 2110-6134

Éditeur

CRAL - Centre de recherche sur les arts et le langage

Référence électronique

Myrtille Picaud et Mathilde Provansal, « Des cheffes orchestrent le travail. Un regard

sociomusicologique », Transposition [En ligne], 5 | 2015, mis en ligne le 01 décembre 2015, consulté le

31 juillet 2019. URL : http://journals.openedition.org/transposition/1241 ; DOI : 10.4000/

transposition.1241

Ce document a été généré automatiquement le 31 juillet 2019.

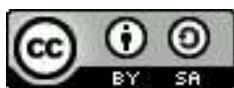

La revue Transposition est mise à disposition selon les termes de la Licence Creative Commons Attribution - Partage dans les Mêmes Conditions 4.0 International. 


\title{
Des cheffes orchestrent le travail. Un regard sociomusicologique
}

\author{
Entretien avec Hyacinthe Ravet
}

Myrtille Picaud et Mathilde Provansal

\section{Le parcours d'une sociomusicologue}

\begin{abstract}
Myrtille Picaud et Mathilde Provansal: Comment s'est construite votre carrière de chercheure à l'intersection entre plusieurs disciplines ? Comment ce parcours spécifique oriente-t-il le rapport aux objets que vous étudiez?
\end{abstract}

Hyacinthe Ravet : Mon parcours associe une formation de musicienne, de sociologue et de musicologue. Allier ces différents types de formation a été réellement important dans l'élaboration de ma réflexion. Lors du choix de mon sujet de recherche en Maîtrise, une observation de mon enseignant de clarinette d'alors, également musicien dans un orchestre professionnel, m'a intriguée. Nous avions de nombreuses discussions à propos du milieu de la musique. Il a remarqué : "Tu sais qu'il n'y a aucune femme clarinettiste dans les orchestres parisiens? Et même en France, il y en a peut-être une ou deux... " Cela aurait pu rester à l'état d'anecdote, mais c'est devenu une vraie question de recherche. J'entretenais alors une double passion pour la pratique instrumentale et pour ce qui fait que les gens vivent, construisent, agissent... et font de la musique ensemble. Or lorsque j'ai commencé à apprendre à jouer de la clarinette, nous étions plus de filles que de garçons parmi les élèves. Le constat de cet enseignant/ musicien n'a donc pas manqué de m'interpeller. L'aiguillon de la réflexion sociologique m'a conduite à me demander s'il ne se jouait pas là un système de reproduction, où les femmes n'arriveraient pas à devenir professionnelles alors qu'elles sont présentes lors de l'apprentissage. Je me suis souvenu des conseils que mon tout premier professeur d'instrument dispensait aux une's et aux autres, au moment des choix de carrière. Suivant sa vision des choses, mieux valait s'orienter vers d'autres études supérieures que la clarinette lorsqu'on était une femme, car la musique était un milieu «trop masculin ", qui n'était pas fait pour elles. J'ai retrouvé la trace de l'une des pionnières en clarinette, qui était passée par sa classe au Conservatoire. Il racontait qu'elle avait eu 
les pires difficultés à devenir professionnelle et qu'elle était finalement entrée dans un orchestre militaire, ce qu'elle m'a confirmé. Alors que je pensais choisir un sujet de recherche à la croisée de la sociologie et de l'économie - les deux disciplines que j'étudiais à cette époque-là en parallèle à mes études musicales -, j'ai finalement troqué les études d'économie contre celles de musicologie. Je voulais me former aux outils de cette discipline afin de mieux appréhender les phénomènes musicaux.

Mais forger une pensée et surtout construire un parcours à l'intersection entre plusieurs disciplines n'est pas chose facile. Par exemple, comment constituer un jury de thèse? Le problème se pose aussi bien sûr au moment des recrutements: dans un contexte de pénurie de postes, on peut aisément vous renvoyer vers l'autre discipline. À présent, la question concerne les étudiante's que je forme. Ce sont principalement des étudiante's en musicologie, intéressée's par l'approche sociologique. L'enjeu est donc de parvenir à acquérir et à tirer parti des outils d'une autre discipline - la sociologie pour approfondir des recherches menées à partir de leur connaissance et de leur pratique de la musique. Ce double ancrage disciplinaire nourrit mes travaux de recherche depuis bientôt une vingtaine d'années ${ }^{1}$. Pour moi, il s'agit de faire de la sociologie de la musique qui soit vraiment à l'écoute du matériau musical lui-même. Si l'on veut atteindre un degré fin de compréhension des phénomènes musicaux, il faut saisir la spécificité de cette activité artistique, de cette forme d'expression. Développer une sociologie de la musique à l'écoute de la musique et à la croisée des deux disciplines enrichit autant la réflexion sociologique que musicologique. Lorsque l'on mobilise les outils analytiques et que l'on plonge au cœur du matériau musical, on peut même parler de sociomusicologie. Dans mon ouvrage sur le travail entre cheffe's et musicien'ne-s qui construisent ensemble une interprétation musicale ${ }^{2}$, je m'appuie sur une connaissance intime du matériau musical, des œuvres, tout autant que sur ma propre pratique orchestrale. Je me sers de la partition comme support d'annotation de mes observations ethnographiques, en faisant le lien entre le texte écrit, le produit sonore, les indications verbales ainsi que les réactions corporelles des participantees. L'analyse musicale est ainsi mise au service d'une compréhension d'un matériau où social et musical sont totalement inextricables.

M. Pi. et M. Pr. : Dans votre mémoire de Maîtrise puis dans votre thèse, vous travaillez les questions de genre. Comment cette entrée particulière dans le monde musical a-t-elle été appréciée?

H. R. : Lorsque j'ai commencé à travailler, en sociologie, sur les questions de genre à propos de musique ${ }^{3}$, certain'e's m'ont quand même un peu regardée d'un air de dire « tu n'as pas grand chose à faire de ta vie »! Les questions de genre représentaient alors un angle mort des recherches sociologiques sur le travail artistique et le domaine de la création. Pourtant, il existait déjà une Histoire des femmes, des travaux historiographiques et épistémologiques sur les créatrices. En sociologie, tout un courant d'analyse des rapports sociaux de sexe dans le travail et la société se développait depuis les années 1970-1980. Lorsque je me suis penchée sur ces questions, au début des années 1990, c'était donc une orientation en pleine émergence: la Conférence de Pékin ${ }^{4}$, les travaux de Margaret Maruani, de Catherine Marry et de bien d'autres éclairaient la réflexion ${ }^{5}$. Mais, de manière générale, les questions de genre n'étaient pas encore un domaine de recherche qui paraissait incontournable en sociologie. D'ailleurs on ne parlait pas de "genre", mais de "rapports sociaux de sexe ». Dans l'Université, une place avait été faite à ces questions par endroits, mais 
dans beaucoup d'autres lieux ce n'était pas les thématiques centrales sur lesquelles on travaillait. Donc combiner art, musique, et genre... cela apparaissait plutôt comme quelque chose de tout à fait exotique ! La musicologie française ne s'était, quant à elle, pas encore intéressée à ces approches. La musicologie américaine nourrie des Cultural Studies - celle qui était qualifiée de New Musicology en particulier - rencontrait de nombreux détracteurs en France. La musicologie française ne s'est en définitive ouverte aux questions de genre que depuis une dizaine d'années seulement. Avec des collègues musicologues et historien'ne's, nous avons ainsi créé le Cercle de Recherche Interdisciplinaire sur les Musiciennes (CREIM) ${ }^{6}$ qui tente de développer ce domaine de recherche sous un angle pluridisciplinaire.

Indice et corolaire du peu de cas que l'on faisait alors de ce domaine, il n'existait pas de données sexuées à propos de la musique lorsque j'ai commencé à travailler sur l'accès et la place des femmes dans les orchestres. Aucun travail systématique ne permettait de s'en faire une idée précise. L'étude menée par Xavier Dupuis ${ }^{7}$ et la thèse de Bernard Lehmann ${ }^{8}$ dressaient un premier tableau, mais il n'y avait pas de recensement exhaustif sur l'ensemble des orchestres français pour savoir : qui, quoi, où ? Cela aurait permis, in fine, de préciser quels types de poste étaient attribués aux femmes. Les sujets peu balisés, pour lesquels il n'y a pas de données, c'est excitant parce que tout est à faire! Depuis plusieurs années, ce type de problématique et de questionnement a acquis une certaine légitimité en sociologie. Mais cela ne signifie pas non plus que tout le monde considère que ce soit important. En matière de genre, rien n'est jamais complètement acquis...

M. Pi. et M. Pr. : Comment a émergé la figure du ou de la cheffe d'orchestre comme objet de recherche?

H. R. : Après avoir travaillé sur les clarinettistes, la question de savoir comment on devient musicienne, ou pas, quel que soit l'instrument pratiqué, m'a occupée. Je me suis intéressée à l'accès à une profession, celle de musicien-ne des orchestres permanents, dont les contours peuvent être cernés précisément puisque ces musicien-ne's sont recrutée'es par auditions sur des postes officiellement mis au concours. L'accès s'entend ici à la fois d'un point de vue historique ainsi que de celui des gate-keepers ${ }^{9}$ et des droits d'entrée ${ }^{10}$. J'ai ainsi essayé de comprendre la construction des carrières et des trajectoires, les relations entre des pratiques et des itinéraires, la manière dont on se représente un instrument, s'il est «masculin» ou "féminin», ce qui fait qu'on le choisit ou pas, et ce que « choisir » veut dire. L'articulation entre ces deux dimensions, pratiques et représentations, a toujours été centrale dans ma réflexion. Donc, j'ai commencé par les musicien'ne's d'orchestre. La figure du chef s'est imposée au fil de l'enquête, omniprésente, en contrepoint. Dans ma thèse, j'ai mêlé différents outils d'enquête. J'ai travaillé à partir de données historiques et de données d'archives pour contextualiser l'accès des femmes aux professions musicales et tenter de le quantifier. J'ai réalisé des entretiens approfondis pour analyser la construction et le déroulement des carrières. J'ai aussi mobilisé un questionnaire à destination des musicien.ne's de plusieurs orchestres; je leur transmettais à l'occasion de sessions de travail et de concerts que j'observais à leurs côtés. Ces observations du travail orchestral nourrissaient la pensée, mais n'étaient pas le point de mire de mon investigation. Elles représentaient un matériau de terrain très dense, à exploiter par la suite, dans lequel la figure du chef est apparue incontournable. 
Après ma thès $\mathrm{e}^{11}$, j'ai également rencontré un certain nombre de cheffes d'orchestre femmes, en particulier Claire Gibault ${ }^{12}$ et Laurence Equilbey ${ }^{13}$. J'ai donc commencé à faire de nouvelles observations systématiques, du côté des cheffe's cette fois. Celles-ci se focalisent sur le travail collectif mené par les participante-s, à partir d'une question d'apparence simple : comment se construit une interprétation musicale? Les questions de genre ne constituent pas la problématique initiale, mais le genre y est appréhendé comme un analyseur de la pratique orchestrale: la présence de cheffes dérange l'ordonnancement classique et révèle des attentes quant à la fonction du chef et au travail collectif.

Lorsque j'ai débuté ces nouvelles observations, notamment auprès de cheffes, je souhaitais articuler le point de vue des musicien-ne's à celui des cheffés. Une relation de longue durée s'est installée avec plusieurs cheffes. Pour certaines, j'observe toujours leur travail et elles me sollicitent parfois pour mon expertise dans le domaine musical. A contrario, lorsque j'avais observé le travail du côté des musicien-ne·s, l'enjeu était clairement de ne pas être perçue comme étant du côté des cheffés ou venant de l'administration. Il n'est pas simple de pouvoir tenir les deux ensemble - musicien'ne's et cheffe's - au cours de l'enquête, comme je l'explique dans mon ouvrage L'orchestre au travail $^{14}$.

M. Pi. et M. Pr. : Quelle a été votre démarche d'enquête dans cet ouvrage précisément, à partir de votre double positionnement de sociologue et de musicologue?

H. R. : Ce livre est le fruit de nombreuses années d'observation au cours desquelles j'ai assisté à des répétitions, des concerts et des séances d'enregistrement, mais aussi dialogué de manière approfondie avec les cheffe's observée's. Plusieurs recherches ont examiné la profession et le métier de musicienne d'orchestre. En outre, Bernard Lehmann a écrit un chapitre sur la répétition ${ }^{15}$; les travaux de Robert R. Faulkner ${ }^{16}$ ainsi qu'un article de Pierre François ${ }^{17}$ examinent aussi les interactions au sein de l'orchestre. Mais finalement, l'activité artistique de l'orchestre demeurait peu documentée de manière fine et intensive. Aucune recherche ne décortiquait en détail ce qui se joue dans le travail musical collectif au quotidien. Une telle activité peut paraître bien connue : le/la cheffe dirige; il/elle choisit un programme; il suffit aux autres - les musicien'ne's - de jouer ce qui est écrit sur la partition. Pourtant, il s'agit d'une fausse évidence. De nombreux éléments implicites interviennent dans l'action, qui sont rarement explicités verbalement. Cela relève notamment du fait que devenir musicien-ne d'orchestre exige des années d'apprentissage instrumental, puis d'apprentissage au sein du collectif. Les choses ne sont pas expliquées; elles sont vécues, expérimentées par la pratique et extrêmement corporelles, aussi bien pour le/ la cheffe que les musicien-ne-s. Lors d'une répétition, beaucoup d'échanges passent par le corps, le regard, l'écoute, au-delà des mots échangés...

Ainsi, mon travail s'est appuyé de manière importante sur l'observation ethnographique de plusieurs orchestres, en lien avec plusieurs cheffe's parmi lesquelle-s Claudio Abbado ${ }^{18}$, Laurence Equilbey, Claire Gibault et Debora Waldman ${ }^{19}$. Outre la tenue d'un journal d'enquête, j'ai mobilisé les partitions pour annoter mes observations : telle remarque à tel moment $\mathrm{du} / \mathrm{de}$ la chef, telle interpellation d'un'e musicien-ne, ce que j'entendais de la réalisation obtenue. Les partitions annotées des cheffe's m'intéressaient également. Cela permet de voir ce qu'ils/elles projettent, mais aussi de les faire parler de leur vision de l'interprétation, de la manière dont ils/elles 
s'adaptent aux musicien-ne's et, éventuellement, à leurs propositions ainsi qu'au travail produit par le collectif. Finalement, pour rendre dicible toutes ces choses que l'ethnographie permet de saisir in situ, au cœur même de l'action, j'ai noté qui le/la cheffe regarde, sur qui il/elle s'appuie, à quel moment ; mais aussi les types de gestes et de déplacements. Il m'importait d'examiner les interactions au travers des indications verbales, y compris les onomatopées musicales très souvent employées, mais aussi de l'activité corporelle. Il s'agit vraiment d'interactions socio-musicales. Telle remarque d'articulation instrumentale et de conduite de la phrase musicale peut relever à la fois de l'interprétation du texte musical, mais aussi de la réaction à la manière de travailler des musicien'ne's, liée aux rapports de pouvoir qui se jouent au sein de l'orchestre. Social et musical sont intimement mêlés et totalement indissociables. On peut dire qu'il s'agit véritablement d'une recherche sociomusicologique, ou musicosociologique si l'on préfère! L'annotation des partitions ainsi que la perception auditive construisent, avec l'observation, un matériau très dense et riche, permettant de caractériser le type d'action, les situations de coopération et de conflit. À partir de ce matériau et de son analyse, j'ai établi une typologie des actions et des modes de relation avec les musicien'ne's, ce qui permet de décliner les modes d'autorité des cheffe's, faisant apparaître combien il s'agit d'une autorité négociée entre les divers participant·•·s. On saisit également de cette manière la sédimentation de l'activité musicale, son élaboration dans le temps, en fonction du temps même de la vie du groupe.

Certaine's collègues expérimentent ce type de recherche, par immersion, en musicologie lorsqu'ils/elles s'attachent à analyser les processus créateurs ${ }^{20}$. La démarche ethnographique peut alors être mobilisée ou une autre forme d'observation qui passe par le fait de remettre en situation de création; par exemple, à propos de compositeurs afin de comprendre empiriquement le travail d'écriture ${ }^{21}$. Cependant, l'activité éminemment collective de l'orchestre me paraît complexifier l'analyse, car de nombreux paramètres et acteurs entrent en jeu.

Or, tenter de cerner l'apport de chacun'e des participantees à l'action conduit à montrer que les processus d'interprétation reposent sur une "créativité partagée ». Dans l'action, des « relais » jouent un rôle essentiel entre le/la cheffe et les musicien'ne·s, et vice versa. Par ailleurs, les musicien-ne's s'autorégulent entre eux/elles, à l'intérieur d'un même pupitre ou de pupitre à pupitre. Des phénomènes d'intervention spontanée de la part des musicien'ne's, qui ne réagissent pas uniquement aux demandes du/de la cheffe, s'observent. Finalement, on en vient à palper empiriquement des phénomènes de coopération qui demandent un engagement physique et artistique des participante-s afin d'élaborer une interprétation. Mon travail de recherche a donc consisté à décliner de manière concrète à la fois l'« autorité négociée » et la «créativité partagée » qui caractérisent la musique en-train-de-se-faire dans un tel ensemble. Analyser comment se construit une interprétation musicale dans le contexte du travail orchestral, en décrivant finement, en décomposant et en typifiant ce type d'action, permet de mieux comprendre les processus créateurs éminemment collectifs en art.

\section{Être chefffe aujourd'hui : données et perspectives}


M. Pi. et M. Pr. : Vous avez évoqué des personnes qui dirigent des orchestres, telles que Claire Gibault et Claudio Abbado. Mais qui sont les chef·fes aujourd'hui ? Et comment devient-on chefffe?

H. R. : Vaste question! Il y a plusieurs manières de devenir cheffe d'orchestre. Par ailleurs, on peut être formé en direction d'orchestre sans devenir cheffe d'orchestre. L'accès à une telle formation fournit cependant un indicateur de l'entrée dans une profession. Il existe une classe de direction d'orchestre au Conservatoire National Supérieur de Musique et de Danse (CNSMD) de Paris et une classe de direction de chœur au Conservatoire National Supérieur de Musique et de Danse de Lyon. Les proportions d'hommes et de femmes y sont tout à fait différentes. Cela renvoie notamment à la distinction entre instruments et voix, car les chœurs sont dirigés de longue date par des femmes. Dès l'époque médiévale, dans les couvents, des femmes dirigeaient la musique vocale et cette tradition a perduré avec des ensembles vocaux composés uniquement de femmes mais aussi, plus tard, avec des ensembles mixtes comportant parfois des femmes à la direction. Entre 1985 et 201422, 14 femmes sur 35 lauréate's ont été diplômées du CNSMD de Lyon en direction de chœur, soit une proportion de $40 \%$. En revanche, il y a beaucoup moins de lauréates en direction d'orchestre. L'ouverture de la classe de direction d'orchestre a eu lieu en 1914 au CNSMD de Paris. Ce n'est qu'en 1952 qu'une première femme y est diplômée. De 1914 à 2014, 10 femmes ont reçu un prix de direction d'orchestre contre 153 hommes, soit une proportion de 6,5\% de femmes diplômées. Parmi les pionnières dans le domaine, on retient le nom de Claire Gibault, troisième diplômée en 1969. On note une accélération relative depuis les années 2000, avec quatre diplômées entre 2005 et aujourd'hui.

Certaine's sont donc formée's par le CNSMD de Paris, en classe de direction d'orchestre. Mais beaucoup apprennent le métier sur le tas ou par des master class ou des stages auprès de chefffe's. Il n'existe pas une seule manière ou un diplôme unique pour devenir cheffe. Les réseaux aussi sont très importants, les personnes avec lesquelles vous travaillez, dont vous êtes l'assistantee, les personnes que vous remplacez au pied levé lors d'une répétition. Il existe également un star system de la direction d'orchestre. Les plus hautes positions sont d'envergure internationale: certaine's cheffe's occupent parfois des positions dans deux ou trois orchestres de renommée mondiale situés aux quatre coins de la planète. Comme pour tout système starifié, il est très difficile d'arriver à ces plus hauts niveaux, pour les hommes comme pour les femmes. En parallèle, il existe un marché plutôt local et national de la direction d'orchestre. De manière schématique, on peut dire que l'on compte quelques élu'e's pour les plus hauts postes et une myriade d'individus pluriactifs qui sont cheffe's d'orchestre ou cheffe's de chœur tout en occupant notamment des postes d'enseignantee's.

M. Pi. et M. Pr.: Quelles sont les proportions d'hommes et de femmes à ces postes de chef.fe?

H. R. : En France, les 30 orchestres permanents représentent un modèle spécifique d'organisation au sein de l'activité artistique. Leurs membres sont en contrat à durée indéterminée, alors que la plupart des musicienne's comme d'autres artistes interprètes sont intermittente's ou cumulent plusieurs emplois. Actuellement, il n'y a plus aucune femme à la direction de l'un de ces orchestres permanents ${ }^{23}$. Trois femmes seulement ont un jour occupé un poste de directeur artistique d'un orchestre permanent français. La dernière était Susanna Mälkki ${ }^{24}$, qui a dirigé l'Ensemble intercontemporain de 2006 à 2013. Il s'agit d'un ensemble assez singulier, spécialisé 
dans le répertoire de la musique contemporaine, comptant une trentaine de musicien'ne's permanent·es, qui sont tou'te's solistes au sein de l'orchestre ${ }^{25}$. Graziella Contratto $^{26}$, quant à elle, a dirigé l'Orchestre des Pays de Savoie, de 2003 à 2009. Il s'agit d'un orchestre de chambre, composé de 23 musicien-ne's, qui travaille sur un territoire particulier, principalement la Savoie et la Haute-Savoie. Claire Gibault avait été directrice musicale de ce même ensemble de 1976 à 1983. Qu'il s'agisse de l'Ensemble intercontemporain ou de l'Orchestre des Pays de Savoie, ces ensembles ne sont pas des orchestres symphoniques, comme l'Orchestre National des Pays de la Loire ou l'Orchestre de Paris par exemple.

Le constat est un peu différent si l'on s'intéresse aux autres ensembles, les formations professionnelles composées de musicien'ne's intermittent·es, notamment en musique ancienne et en musique contemporaine. Les données de l'Association pour la gestion de la sécurité sociale des auteurs (AGESSA) montrent qu'il y a environ $8 \%$ de femmes parmi les cheffés de ces ensembles. Quant à ceux qui sont subventionnés par le ministère de la Culture, par le biais des DRAC notamment, ils sont de tailles diverses et évoluent au sein de différents genres musicaux, de la musique ancienne à la chanson, en passant par les musiques traditionnelles ou les compagnies de théâtre musical. Il ne s'agit pas non plus d'orchestres symphoniques. Ce sont des ensembles qui emploient des musicien'ne's intermittente's et qui sont repérés comme des ensembles musicaux. Selon les années, il y a entre 15 à $20 \%$ de femmes cheffes ${ }^{27}$. En 2009, 58 femmes dirigeaient ou co-dirigeaient l'un de ces 305 ensembles subventionnés. Un vivier important de cheffes existe donc, contrairement à ce que l'on entend dire parfois. L'objet de mes investigations, c'est donc aussi de tenter d'établir un constat chiffré et de remonter dans le temps, afin de dresser un état des lieux de la situation et ensuite de tenter d'expliquer cette dernière. Mais il faudrait systématiser la quête de données régulières à un niveau institutionnel. C'est ce à quoi s'emploie la Société des auteurs et des compositeurs dramatiques (SACD) en lien avec le Laboratoire de l'Égalité et le Mouvement H/F pour l'égalité entre femmes et hommes dans l'art et la culture ${ }^{28}$. De telles données chiffrées sont indispensables pour lutter contre le déni de processus discriminatoires, même au sein d'un domaine comme la culture et les arts, et peut-être plus encore en musique qu'ailleurs...

Finalement, la question des cheffes est semblable à celle des compositrices: on a souvent pensé qu'il n'y en avait pas, mais différentes recherches ont montré qu'il en existait bien plus qu'on ne l'imagine, pour certaines extrêmement renommées à leur époque $^{29}$. Ainsi, on retrouve dans le passé des traces de cheffes d'orchestre très reconnues, comme Nadia Boulanger ${ }^{30}$ par exemple. Elle s'est imposée comme cheffe d'orchestre de renom international et a été la première à diriger, en tant que cheffe invitée, des orchestres symphoniques de premier rang, dès les années 1930, tels que le Royal Philharmonic Orchestra, le Boston Symphony Orchestra, l'Orchestre de Philadelphie ou encore le New York Philharmonic Orchestra. D'autres auparavant avaient eu une activité importante de direction au sein des Salons, comme Fanny Mendelssohn ${ }^{31}$.

De manière plus générale, et comme pour les musiciennes interprètes, des phénomènes de ségrégation caractérisent la position des cheffes dans la profession : une ségrégation verticale, car les femmes dirigent des ensembles spécifiques, qu'elles ont le plus souvent (co)fondés, de taille plus modeste que les orchestres symphoniques permanents; une 
ségrégation horizontale, car elles dirigent plutôt certains types de répertoires, plus souvent en musique ancienne ou encore lié au chant.

M. Pi. et M. Pr. : Et quels sont les facteurs permettant d'expliquer la faible féminisation de cette profession?

H. R. : Cela renvoie d'abord aux modalités historiques de l'émergence de la figure du chef d'orchestre. Cette figure se consolide avec la symphonie romantique. Il existait auparavant d'autres formes de direction. Jean-Baptiste Lully, par exemple, dirigeait ses tragédies musicales du bâton. Les ensembles baroques étaient souvent dirigés du pupitre par le premier violon. La figure du chef responsable de l'interprétation de l'œuvre, au sens d'une nouvelle naissance de l'œuvre, émerge et s'impose progressivement au cours du XIX ${ }^{e}$ siècle. Hector Berlioz, notamment, a théorisé le rôle majeur du chef qui dirige un ensemble conséquent d'instrumentistes. Le chef sur un podium, dos au public, face à l'œuvre et à un orchestre composé uniquement d'instrumentistes hommes alors, progressivement considéré comme une sorte de supra-interprète, un co-créateur ou un recréateur, est une figure qui concentre beaucoup d'enjeux symboliques. Le régime « vocationnel » de l'artiste qui s'impose dans le même temps en se virilisant consolide cette figure, qui peut apparaître comme une sorte de paradigme de la figure du génie en musique. Progressivement, le chef devient une star, au point même parfois d'effacer ou de concurrencer la figure du compositeur. Durant le XIX ${ }^{e}$ siècle bourgeois, les règles de décence ne permettaient pas aux femmes ou seulement de manière exceptionnelle - de s'exposer dans de telles situations de pouvoir ni de prétendre accéder aux fonctions démiurgiques.

En second lieu, l'accès des femmes aux orchestres professionnels n'a pas été simple. En effet, elles n'étaient pas acceptées dans les ensembles qui se sont constitués au cours du $\mathrm{XIX}^{\mathrm{e}}$ siècle, sauf exceptions : on trouvait parfois une harpiste. Lorsque le Conservatoire de Paris (futur Conservatoire National Supérieur de Musique et de Danse de Paris) ouvre à la fin du XVIII ${ }^{\mathrm{e}}$ siècle, il s'agit d'une institution mixte. Les femmes sont admises au piano, au chant et au solfège, dans des classes qui leur sont dédiées. Progressivement, au cours du XIX ${ }^{\mathrm{e}}$ siècle, elles accèdent aux classes d'instrument à cordes puis, au début du XX $\mathrm{XX}^{\mathrm{e}}$ siècle, à certaines classes d'instrument à vent. Des diplômées de l'institution revendiquent ensuite l'accès aux professions musicales, non seulement comme enseignantes, mais aussi comme instrumentistes d'orchestre. Des polémiques éclatent. Certaines parviennent à se faire admettre dans des ensembles, mais l'accès à la direction leur demeure refusé. La fin du XIX et le début du $\mathrm{XX}^{\mathrm{e}}$ siècle ont également vu la création d'orchestres uniquement féminins. Jane Evrard ${ }^{32}$ a dirigé l'Orchestre féminin de Paris, qu'elle avait fondé en 1930, un ensemble constitué de 25 musiciennes d'orchestre qui a tourné en Europe jusqu'à la Seconde Guerre mondiale. Des compositeurs comme Arthur Honegger, André Jolivet ou Albert Roussel ont composé des œuvres pour cet ensemble. Ici, la cheffe pouvait être une femme, car il s'agissait de diriger des femmes.

Aujourd'hui encore, il ne va pas de soi qu'une femme puisse diriger une assemblée d'hommes. Un soupçon pèse toujours sur la cheffe : va-t-elle réussir à soumettre une assemblée d'hommes à son autorité ? Et comment? Les femmes ont aussi souvent été perçues comme perturbant l'entre-soi dans les orchestres. Et ces arguments se retrouvent parfois dans la bouche de jeunes musicien'ne's aujourd'hui. Les femmes instrumentistes ne sont pas toujours et pas forcément les plus favorables à l'arrivée des femmes au pupitre, parce que longtemps, on les a mises en concurrence, femme contre 
femme. Les cheffes évoquent aussi cette concurrence. Elles ne bénéficient pas de réseaux au même titre que leurs collègues hommes, le réseau entre elles n'étant pas encore vraiment constitué.

De plus, la direction offre une mise en scène du pouvoir très forte, avec le/la cheffe sur le podium, tenant la baguette dans laquelle on peut voir un attribut phallique, et formant des gestes qui parfois « percent » ou " percutent ». L'orchestre symphonique se présente quant à lui comme l'archétype d'un modèle de société bourgeois. Un microcosme qui reflèterait la vision d'une société harmonieuse, où chacune serait bien à sa place, selon son rang, à son pupitre. Chacune intervient à son tour, quand on lui donne la parole. Le chef incarne ici la figure du pater familias qui guide l'ensemble. En outre, les représentations usuelles de la figure du chef s'incarnent en deux extrêmes. Soit celui-ci dirige tout et c'est le maitre du monde, comme l'écrit Elias Canetti ${ }^{33}$. Soit, c'est l'histrion qui s'agite au pupitre, superflu, car il est finalement le seul qui ne joue pas d'un instrument. Le chef se distingue du chorégraphe ou du metteur en scène qui sont rarement présents sur la scène. S'agissant des femmes, la question de l'indécence du corps en représentation intervient, ainsi que celle de sa "puissance ». Il y a la dimension physique, avec les caractéristiques supposées de mollesse et de faiblesse des femmes, mais aussi la dimension créatrice, selon cette alternative rebattue (s'il en est !) entre procréation et création. Ces commentaires se retrouvent au fil des articles de presse, dans le passé, mais aussi lors de déclarations publiques très récentes d'acteurs du milieu musical.

Ainsi, les critiques soulignent parfois davantage l'apparence physique de la cheffe en scène que ce qu'elle fait réellement, son travail précisément. Les chaussures à talons de Simone Young ${ }^{34}$ sont presque toujours évoquées. Ou alors, les articles présentent chacune des cheffes comme une exception : malgré tout, elle reste féminine, clamentils, alors que - par ailleurs - elle dirige " comme un homme", s'exclament d'autres. C'est censé d'ailleurs être le meilleur compliment adressé à une cheffe d'orchestre ou à une compositrice : vous composez, vous écrivez, vous dirigez « comme un homme »... Leur vie familiale fait souvent l'objet de commentaires. On ne trouve pas d'équivalent à propos des chefs hommes, tout particulièrement l'insistance sur la figure exceptionnelle de la femme-cheffe. Ainsi que le signale Juliette Rennes à propos des professions de prestige ${ }^{35}$, l'aspect extra-ordinaire conduit à s'intéresser au paraître (du côté du «féminin ») plutôt qu'à l'être ou à l'agir (du côté du «masculin»), et ici précisément au type d'interprétation musicale que les cheffes construisent.

De manière plus implicite, on retrouve aujourd'hui ces arguments supposant une moindre force physique et morale des femmes sous la forme de doutes émis à l'égard des choix de programmation ou d'interprétation ou de «conseils» arguant, par exemple, que les femmes seraient plus douées pour diriger de "petites" œuvres. "Jusqu'à Mozart, ça va; pour Beethoven, cela dépend des symphonies, on va peut-être s'arrêter à la cinquième! ", disent en substance certaines des enquêtées à propos des réactions qu'elles rencontrent. Ce n'est pas qu'anecdotique. Par exemple, Simone Young, qui dirige l'Opéra de Hambourg, ou Marin Alsop ${ }^{36}$ qui dirige l'Orchestre symphonique de Baltimore, construisent chacune une carrière internationale. Mais elles font face à des critiques sur le fait qu'elles dirigent de manière trop « masculine ». $\mathrm{Ou}$ encore doivent-elles lutter pour diriger le répertoire symphonique canonique. Simone Young a dû batailler auprès de l'administration de son orchestre pour pouvoir enregistrer les symphonies d'Anton Bruckner: «La symphonie postromantique dans 
tous ses débordements, c'était trop fort!» On considérait qu'elle n'avait pas la puissance pour diriger ou enregistrer ces œuvres. Elle en a finalement enregistré l'intégrale. D'autres ont parfois bataillé juridiquement avec l'orchestre qu'elles étaient amenées à diriger, comme Marin Alsop lorsqu'elle a été nommée à Baltimore et que l'orchestre a contesté sa nomination. Celle-ci a finalement réussi à diriger l'ensemble, mais une ou deux saisons après sa nomination effective. La critique musicale s'est faite ensuite très élogieuse. On a dit qu'elle renouvelait l'orchestre, qu'elle le rendait plus dynamique. Depuis, les musicien-ne's de l'orchestre lui ont demandé de reconduire son contrat; elle y reste jusqu'en 2021. Les résistances peuvent venir des musicien-ne's ou de l'administration, et ces phénomènes illustrent la prégnance de cette figure d'un chef recréateur, qui demeure blanche, occidentalo-centrée et masculine dans les représentations qui lui sont attachées.

M. Pi. et M. Pr. : La présence d'une femme cheffe peut-elle entraîner une féminisation de l'orchestre?

H. R. : Il est difficile de répondre à cette question. Tout d'abord, en France, les cheffe's ne choisissent pas directement les musicien'ne's, sauf quand il s'agit de leur propre ensemble où ils/elles invitent tel ou telle musicien'ne-s à les rejoindre. Les recrutements des musicien'ne's des orchestres permanents se font par concours. Et la question qui se pose effectivement de manière cruciale est celle de la composition des jurys où des efforts demeurent à faire pour les rendre paritaires. De plus, les cheffes ont été jusqu'à présent très peu nombreuses à la tête des orchestres professionnels permanents. Si l'on prend l'exemple de la direction par Susanna Mälkki de l'Ensemble intercontemporain pendant quelques années, elle n'a pas sensiblement modifié le recrutement en faveur des femmes. Toutefois, le nombre de recrutements comptabilisés dans les données disponibles est faible, donc il n'est pas possible de se prononcer de manière définitive. Concernant les orchestres intermittents, une cheffe comme Claire Gibault fait explicitement attention à recruter des femmes dans les cuivres, notamment aux cors. D'autres cheffes, dans la musique ancienne ou la musique contemporaine, font également appel à des musiciennes, d'autant plus que le recrutement s'opère souvent par cooptation et par interconnaissance, plus rarement par audition. Cela peut donc contribuer à une féminisation, à la différence des orchestres permanents, si les cheffes sont sensibilisées à ces questions.

Avec des collègues, nous avons fait une enquête sur les effets de la présence du "paravent » lors des procédures de recrutement, c'est-à-dire lors des auditions à l'aveugle dans les orchestres. La problématique au cœur de notre premier rapport de recherche ${ }^{37}$ est celle de l'influence de ce type de procédure sur l'accès des femmes aux orchestres : peut-on mesurer de manière systématique que la présence d'un paravent ou d'un rideau masquant le sexe $\mathrm{du} / \mathrm{de}$ la candidate permet à davantage de femmes de passer les différents tours des concours et in fine d'être plus souvent recrutées, comme de nombreuses pionnières d'orchestre l'ont racontée ${ }^{38}$ ? Les données collectées ne permettent pas de conclure en l'état sur un effet positif ou négatif de ce type de dispositif. Mais l'étude montre cependant qu'avec ou sans paravent ${ }^{39}$, un phénomène d'élimination progressive des musiciennes au cours des premier et deuxième tours, voire troisième lorsqu'il existe, est observable lors du recrutement, et ce sans ambiguïté.

La question de la féminisation peut également être posée par rapport à la programmation. La présence d'une cheffe à la tête d'un ensemble ouvre-t-elle la 
programmation aux œuvres des compositrices, dont on sait qu'elle reste ténue de manière générale en France ${ }^{40}$ ? Certaines cheffes ont longtemps été réticentes à diriger des œuvres de compositrices. Une fois de plus, elles se trouvaient prises dans des questionnements contradictoires : ce répertoire leur est souvent proposé parce qu'elles sont femmes alors qu'il s'agit effectivement d'un répertoire qu'il serait nécessaire de faire mieux connaître. Claire Gibault explique qu'on lui a demandé, par exemple, de diriger une œuvre de Fanny Mendelssohn qu'elle ne trouvait pas intéressante par le passé. Cela s'est donc avéré compliqué pour elle, car elle ne souhaitait pas défendre cette œuvre-là, mais d'autres, comme elle le fait aujourd'hui avec son ensemble, le Paris Mozart Orchestra. Certaines cheffes voient aussi cela comme l'assignation à une forme de ghetto, qui les enfermerait dans ce type de répertoire et les représentations dépréciatives qui y sont fréquemment attachées : des œuvres de femmes dirigées par des femmes, sous-entendu des œuvres «mineures » dirigées par des cheffes qui n'ont pas la carrure des "grands ». C'est à l'ensemble de ces idées reçues qu'il est nécessaire de s'attaquer, et la tâche n'est pas mince !

À celles qui souhaitent développer une carrière de cheffe d'orchestre et diriger le répertoire symphonique, il est ainsi souvent d'abord proposé de diriger des œuvres vocales et des œuvres pour enfants. Pour certaines, c'est véritablement un choix. Laurence Equilbey est passée de la direction vocale, apprise en même temps que la direction d'orchestre, à la direction de productions lyriques, notamment à l'Opéra de Rouen. Elle en est arrivé ensuite à diriger de plus en plus des orchestres avec ou sans chœur, d'abord des ensembles de musique ancienne, puis par la suite des ensembles symphoniques permanents en tant que cheffe invitée, avant de créer son propre ensemble instrumental - Insula Orchestra - et de poursuivre son activité de cheffe invitée auprès des orchestres permanents. Cette question se pose toujours aujourd'hui avec les jeunes femmes cheffes, telle que Debora Waldman, à qui les administrations d'orchestre ou de festival proposent de diriger des concerts familiaux, des concerts "jeune public», des concerts "participatifs", des concerts en plein air, ailleurs que dans les grandes salles, et plus rarement des productions symphoniques dans des salles prestigieuses. Bien entendu, ces répertoires et types de concert sont tout à fait intéressants et importants. Cependant, cela les associe prioritairement aux enfants, à la voix : il ne s'agit pas du « grand » répertoire canonique dans la « grande » salle. Debora Waldman a ainsi dirigé La Flûte à chanter à la Philharmonie de Paris au moment de son ouverture, un moment fort donc. « Mais » (avec des guillemets, car il n'y a rien à mes yeux de dépréciatif), il s'agissait d'un concert participatif à destination des familles, qui mobilisait des solistes lyriques et plusieurs instrumentistes (et non un orchestre au complet).

M. Pi. et M. Pr. : Les formations sans chefffe ménagent-elles une plus ou moins grande place aux femmes ? Existe-t-il d'autres formes de direction pouvant faciliter la présence des femmes?

H. R. : Certains ensembles revendiquent effectivement le fait de ne pas être dirigés par une cheffe. Certain'e's musicien-ne's souhaitent travailler sans figure tutélaire qui dirige l'interprétation. Ils mettent en place des stratégies afin de travailler, car ce n'est pas simple de se mettre d'accord pour élaborer une interprétation lorsque vous êtes un groupe de plusieurs instrumentistes. Ce sont essentiellement de petits ensembles dépassant rarement plus d'une trentaine de musicien'ne's, plutôt des orchestres de 
chambre que de grands orchestres, sinon cela devient très difficile de coordonner l'activité.

Certaines formations, comme l'ensemble Orpheus ${ }^{41}$, se dotent très clairement de règles d'action ${ }^{42}$. Après avoir connu des répétitions interminables, les membres ont décidé, par exemple, que celui ou celle qui parle a vraiment quelque chose à dire et à proposer à l'ensemble. Ces ensembles ont pour stratégie de construire des directions collectives, à plusieurs musicien'ne's qui tournent au fil des œuvres ou des sessions de répétitions et de concert. Ces systèmes de direction tournante impliquent de recruter des musicien-ne's qui soient à la fois capables de suivre, mais aussi de décider et de diriger le jeu. Un certain nombre d'ensembles sont constitués de musicien'ne's qui se positionnent explicitement comme n'étant pas de simples exécutante-s. Cela participe de la transformation du métier de musicien-ne d'orchestre et permet de promouvoir une autonomie dans le travail. Les musicien.ne's de ces ensembles dressent ainsi un cadre d'action collectif. C'est toutefois un cadre difficile, voire impossible à mettre en place et à tenir pour les vastes symphonies postromantiques à 130 musicien.ne·s.

M. Pi. et M. Pr. : Quelles sont les stratégies que ces femmes cheffes d'orchestre adoptent pour se maintenir dans la profession?

H.R.: Différentes stratégies de positionnement professionnel s'observent dans le déroulement de la carrière et ne sont pas perçues de la même manière par toutes les cheffes. Par exemple, par la création de son propre ensemble musical. Claire Gibault explique que son passage en politique, en tant que députée européenne, l'a conduite à prendre conscience du fait qu'une des seules manières de diriger pour les femmes était de créer leur propre structure, dans les entreprises comme dans les orchestres. Ayant longtemps attendu d'être nommée comme cheffe d'un ensemble permanent, elle a finalement créé le Paris Mozart Orchestra en 2010, sur le modèle de celui créé à Bologne par Claudio Abbado, auprès duquel elle avait travaillé en tant qu'assistante et qu'elle avait aidé dans la création de cet ensemble. Créer un orchestre de chambre de type intermittent avec une trentaine de musicien'ne's relevait donc véritablement d'une stratégie pour elle afin de pouvoir diriger sur le long terme. Au contraire, Laurence Equilbey ne revendique pas la création d'un ensemble comme une stratégie de femme. Elle a fondé deux ensembles, le chœur de chambre Accentus, en 1992, avec lequel elle a beaucoup travaillé a cappella, puis Insula Orchestra, en 2012, un orchestre intermittent de type " troisième génération » des orchestres baroques. Selon elle, créer un ensemble ici relève davantage d'une nécessité en lien avec la position des cheffés de musique ancienne : ponctuellement invitéees à diriger d'autres ensembles, elles ou ils ne peuvent effectuer un véritable travail de fond sur le son. Le développement d'une carrière de chef passe pour eux ou elles par un lien fort avec leur propre ensemble. Laurence Equilbey ne souhaite donc pas que cette situation soit présentée comme une stratégie menée en tant que femme chef. Pour ma part, il me semble que créer son propre ensemble peut être l'une des stratégies possibles pour les cheffes afin de diriger de manière pérenne, sans que ce soit la seule ni l'unique raison de la création d'un ensemble.

Une autre manière de se maintenir professionnellement peut consister en la création d'un réseau de soutiens. Le travail en tant qu'assistantee en est l'une des étapes, la difficulté étant de ne pas être perçue comme assistantee «à vie » : c'est une situation complexe, pour les hommes comme pour les femmes. Claire Gibault a souvent été présentée, voire perçue, même lorsqu'elle dirigeait ses propres productions, comme 
l'assistante de Claudio Abbado, car elle l'a assisté sur plusieurs productions et ce durant plusieurs années. Par la suite, il a fallu qu'elle s'impose en tant que cheffe pour qu'elle soit appelée à diriger à titre principal, et non en tant qu'assistante.

On observe également d'autres formes de stratégies qui relèvent plutôt d'un travail quotidien effectué avec les musicien-ne's. Certaines femmes cheffes travaillent leur apparence physique, cherchant à se "masculiniser", à faire oublier qu'elles sont des femmes pour mieux endosser les habits du «chef " (entendu ici sous ses connotations viriles). De manière toute différente, d'autres tentent de neutraliser leur apparence et leur positionnement, afin de rendre moins visible le fait qu'il s'agit d'une femme qui dirige, que cela n'apparaisse pas comme une caractéristique essentielle. Certaines composent une identité à double facette, par exemple comme Claire Gibault qui porte une robe-redingote sur pantalon. Ce « costume de travail » associe du « féminin » et du « masculin ». Le corps féminin, sa «féminité » - mais laquelle ? et qu'entend-on par là ? -, n'est pas entièrement nié(e), ni effacé(e), mais il ou elle n'est pas non plus mis(e) en avant comme un attribut central. Selon Claire Gibault, le fait de porter un costume est une manière de montrer que la fonction de direction prédomine sur la personne. Or, aujourd'hui, des amiee's la questionnent à ce propos, en lui demandant pourquoi elle ne s'habille pas de manière plus " féminine » voire " plus sexy » lorsqu'elle dirige, afin de se «mettre en valeur». Depuis peu de temps, en effet, quelques figures de cheffes dirigeant en robe jouent de la "féminité » comme atout pour diriger. Pour Claire Gibault, cela contrevient à l'idée même qu'elle se fait du métier, où il est nécessaire d'oublier la personne, ou plutôt le personnage joué par celle-ci, au bénéfice de la fonction et donc, finalement, de la musique.

Ces questions autour de la visibilité du corps rejoignent celles sur la manière de diriger un ensemble. Se faire accepter par un ensemble en étant une femme est loin d'être simple. Il est nécessaire de composer une autorité acceptable, balancée entre des injonctions ambivalentes et contradictoires. En effet, il faut montrer suffisamment de poigne pour se faire respecter, et donc mettre en scène l'autorité. Cependant, il est souvent reproché aux cheffes d'être trop autoritaires, de sur-jouer une autorité dont elles n'auraient pas hérité de manière "naturelle». Cela constitue un parfait phénomène de double bind, d'injonction paradoxale qui contient une contradiction dans les termes et représente souvent une impasse: si les cheffes ne s'imposent pas assez, cela est perçu comme étant lié à leur statut de femmes ; en tant que femmes, elles n'en seraient pas capables. Mais une revendication un peu forte d'autorité sur un groupe apparaît moins acceptable de la part d'une femme que de celle d'un homme. Négocier une position entre ces deux injonctions, trouver sa propre voie entre ces deux écueils, n'est donc pas aisé.

Il est intéressant de voir que ces questions se traduisent dans la manière même d'organiser le travail au sein des ensembles. Par exemple, la charte du Paris Mozart Orchestra créé par Claire Gibault inclut la parité au cœur des postes à responsabilité, notamment pour ceux de soliste. La vie familiale est prise en compte, par exemple en remboursant le baby-sitting de celles et ceux qui en ont besoin les soirs de concert ou de répétition. Cela n'est pas commun à tous les orchestres. De plus, Claire Gibault se rémunère à même hauteur que les musicien'ne's, ce qui constitue une vraie rupture dans la manière de se positionner en tant que cheffe, le salaire de ces dernier-es étant traditionnellement bien plus élevé. D'une certaine manière, elle se considère comme les 
musicien-ne-s. Cela fait d'ailleurs grincer des dents d'autres cheffe's, qui estiment que cela porte atteinte à la profession et au métier.

Ainsi, le cheffe ne décide pas de tout. C'est d'autant plus vrai aujourd'hui que les musicien'ne-s ne sont plus prête's à accepter une autorité autoritariste «à la Toscanni » ou «à la Karajan ». La formation de musicien-ne d'orchestre est elle aussi en train de changer. Celle-ci n'est plus seulement instrumentale et s'ouvre à de nombreuses autres disciplines, dont les sciences humaines. Les musicien'ne's souhaitent participer davantage aux décisions liées à la vie de l'orchestre, au travail d'interprétation, voire envisagent des casquettes complémentaires à celles d'instrumentiste et - souvent d'enseignante. Plusieurs cheffes y voient une carte à jouer dans ces changements des modes de direction et d'autorité; une nouvelle stratégie possible serait de s'inscrire dans les transformations de la profession en introduisant plus de coopération et de dialogue avec les musicien'ne's.

Différents facteurs permettent donc d'expliquer la faible féminisation de la profession de chef, qui renvoient notamment à l'émergence d'une figure "masculine » du chef d'orchestre et aux représentations sexuées qui continuent de peser sur celle-ci ainsi que sur son activité. D'autres dimensions mériteraient de plus amples développements, ainsi l'examen du croisement entre les différent rapports sociaux (notamment de classe et de « race $»^{43}$, lesquels s'articulent avec le genre), qui éclairent autant les résistances que les mutations en cours dans cette profession et dans l'activité orchestrale.

\section{NOTES}

1. En 2000, Hyacinthe Ravet a soutenu à l'université Paris X-Nanterre une thèse intitulée Les Musiciennes d'orchestre. Interactions entre représentations sociales et itinéraires, qui nourrit en partie l'ouvrage: RAVET, Hyacinthe, Musiciennes. Enquête sur les femmes et la musique, Paris, Éditions Autrement, 2011.

2. RAVET, Hyacinthe, L'Orchestre au travail. Interactions, négociations, coopérations, Paris, Vrin, 2015.

3. Pour un développement plus conséquent sur ces questions, voir RAVET, Hyacinthe, « Le sexe du travail artistique : un genre nouveau ! ", in DEMAZIÈRE, Didier et PARADEISE, Catherine (dir.), Sociologies. Héritages (1960-2010), Rennes, PUR, à paraître en 2015.

4. La Quatrième conférence mondiale sur les femmes s'est tenue sous l'égide de l'ONU à Pékin en 1995. Celle-ci a débouché sur la Déclaration et le Programme d'action de Pékin en faveur de l'autonomisation des femmes, de la promotion des femmes et de l'égalité des sexes.

5. Voir par exemple LAUFER, Jacqueline, MARRY, Catherine, MARUANI, Margaret, Masculin Féminin : questions pour les sciences de l'homme, Paris, PUF, 2001 ; MARUANI Margaret, Femmes, genre et sociétés, l'état des savoirs, Paris, La Découverte, 2005.

6. Le CREIM a été fondé en 2010 par Catherine Deutsch, Florence Launay, Raphaëlle Legrand, Bertrand Porot et Hyacinthe Ravet.

7. DUPUIS, Xavier, Les Musiciens professionnels d'orchestre. Étude d'une profession artistique, Paris, Ministère de la culture et de la francophonie, Département des études et de la perspective, 1993.

8. Repris dans l'ouvrage: LEHMANN, Bernard, L'Orchestre dans tous ses éclats. Ethnographie des formations symphoniques, Paris, La Découverte, 2002. 
9. Les « gardien'ne's du temple » artistique, celles et ceux qui contrôlent l'accès à un univers ou une profession artistique(s).

10. MAUGER, Gérard (dir.), Droits d'entrée. Modalités et conditions d'accès aux univers artistiques, Paris, Éd. de la Maison des sciences de l'homme, 2007.

11. Notamment à l'occasion du colloque Sorbonne-IRCAM en 2002, "L'accès des femmes à l'expression musicale», lors d'une table ronde consacrée aux cheffes, avec claire Gibault, Laurence Equilbey et Nicole Paiement.

12. Née en 1946, au Mans.

13. Née en 1962, à Paris.

14. RAVET, L'Orchestre au travail. Interactions, négociations, coopérations, op. cit.

15. LEHMANN, «La répétition : construction sociale de l'interprétation », in L'Orchestre dans tous ses éclats, op. cit., p. 192-220.

16. Voir, par exemple, FAULKNER, Robert R., «Orchestra Interaction: Some Features of Communication and Authority in an Artistic Organization ", The Sociological Quarterly, vol. 14, $\mathrm{n}^{\circ}$ 2, 1973, p. 147-157 et "Career Concerns and Mobility Motivations of Orchestra Musicians », The Sociological Quarterly, vol. 14, n 3, 1973, p. 334-349.

17. FRANÇOIS, Pierre, «Production, convention et pouvoir : la construction du son des orchestres de musique ancienne », Sociologie du travail, $n^{\circ} 44,2002$, p. 3-19.

18. Né en 1933, à Milan, et mort en 2014, à Bologne.

19. Née en 1977, au Brésil.

20. Voir les colloques « Tracking the Creative Process in Music», dont celui ayant lieu à Paris du 8 au 10 octobre 2015, co-organisé par Nicolas Donin, Hyacinthe Ravet et Jean-François Trubert.

21. Voir les travaux de Nicolas Donin et Jacques Theureau, par exemple: «L'interprétation comme lecture? L'exemple des annotations et commentaires d'une partition par Pierre-André Valade ", Musimédiane, $\mathrm{n}^{\circ}$ 2, 2006 : http://www.musimediane.com/numero2/Donin.html

22. Les premier'e's diplômée's l'ont été en 1985, car le Conservatoire National Supérieur de Musique et de Danse de Lyon a été créé en 1979.

23. Voir le chapitre "Madame, le chef", dans RAVET, L'Orchestre au travail. Interactions, négociations, coopérations, op. cit., d'où est issu l'ensemble des données citées sur les cheffes.

24. Née en 1969, à Helsinki.

25. La plupart des orchestres permanents, en particulier les orchestres symphoniques et lyriques, comportent des postes de "tuttistes", qui sont des musicien'ne's du rang jouant toujours avec les membres de leur pupitre. Ces postes se distinguent donc de ceux de "solistes ", lesquels jouent les voix principales à l'instrument et exercent pour certain'e's des fonctions de cheffe de pupitre.

26. Née en 1966, à Schwytz.

27. Les dernières données disponibles datent de 2009.

28. Voir la quatrième brochure Où sont les femmes? Saison 2015-2016, éditée par la SACD en partenariat avec le Laboratoire de l'Égalité et le Mouvement H/F.

29. Voir, par exemple, LAUNAY, Florence, Les Compositrices en France au XIX ${ }^{e}$ siècle, Paris, Fayard, 2006.

30. Née en 1887, à Paris, où elle est décédée en 1979.

31. Née en 1805, à Hambourg, et morte en 1847, à Berlin.

32. Née en 1893, à Neuilly-Plaisance, et morte en 1984, à Paris.

33. CANETTI, Elias, Masse et puissance [1960], trad. R. Rovini, Paris, Gallimard, 1966.

34. Née en 1961, à Sydney.

35. RENNES, Juliette, Le Mérite et la nature. Une controverse républicaine : l'accès des femmes aux professions de prestige, 1800-1940, Paris, Fayard, 2007.

36. Née en 1956, aux États-Unis. 
37. HATZIPETROU-ANDRONIKOU, Reguina, NOÉ, Chiara, PÉRIVIER, Hélène et RAVET, Hyacinthe, Écouter sans voir. L'impact du paravent sur le recrutement des musicien-ne-s dans orchestres permanents de Paris et d'île-de-France, Paris, IReMus-Université Paris-Sorbonne, Presage-Sciences Po-OFCE, 2015.

38. Cf. RAVET, Musiciennes. Enquête sur les femmes et la musique, op. cit.

39. Dans les données qui ont été collectées et constituées, certains orchestres n'utilisaient pas de paravent au moment de l'étude.

40. La brochure Où sont les femmes? Saison 2014-2015 montre que les principaux orchestres subventionnés ont programmé 11 femmes sur 804 compositeurs, soit $1 \%$ d'entre eux/elles, durant la saison concernée.

41. Il s'agit d'un orchestre de chambre fondé, en 1972, par le violoncelliste Julian Fifer, et basé à New York.

42. Voir, par exemple, KHODVAKOV, Dimitry M., "The Complexity of Trust-Control Relationships in Creative Organizations: Insights from a Qualitative Analysis of a Conductorless Orchestra ", Social Forces, $n^{\circ} 86,2007$, p. 1-22.

43. Le terme est utilisé ici dans son sens sociologique, comme concept permettant d'étudier des rapports de domination fondés sur une naturalisation de l'altérité.

\section{RÉSUMÉS}

Professeure à l'Université Paris-Sorbonne, Hyacinthe Ravet a suivi un parcours mêlant sociologie, musicologie et musique. Elle revient ici sur les spécificités de l'approche sociomusicologique, en termes de méthode et de construction de l'objet, en abordant son analyse du travail orchestral sous l'angle du genre. Après avoir consacré sa thèse de doctorat aux musiciennes, ses recherches récentes se concentrent sur les cheffes d'orchestre. Dans cet entretien, Hyacinthe Ravet expose les voies suivies par ces femmes au sein d'une profession construite au masculin et les processus sociaux menant à sa faible féminisation. Il est également question du travail orchestral réalisé en coopération entre les musicien'ne's et les chef.fe's. L'analyse des pratiques de direction et d'interprétation d'une œuvre permet ainsi d'aborder la question de la musique « en-train-de-sefaire » et de la « créativité partagée ».

Professor at the Paris-Sorbonne University, Hyacinthe Ravet has followed paths that have led her to sociology, musicology and music. Here, she explains the specificities of a socio-musicological approach, illustrating how it influenced the construction of her research topic about orchestral work seen through a gendered lens, as well as the methodology she used. Her doctoral dissertation was centered on women musicians, and she currently works on female conductors. In this interview, Hyacinthe Ravet exposes how women have come to this profession, considered as particularly masculine, and the social mechanisms that lead to its low feminization. She also talks about the orchestral work in which conductors and musicians participate. The analysis of the practices of conducting and interpreting the music enables to unearth the way music is done and made, as well as processes of "shared creativity." 
INDEX

Keywords : gender, artistic professions, work, musical creation, musicology, sociology.

Mots-clés : genre, professions artistiques, travail, création musicale, musicologie, sociologie.

\section{AUTEURS}

\section{MYRTILLE PICAUD}

Attachée Temporaire d'Enseignement et de Recherche (ATER) à l'Université Paris 1 PanthéonSorbonne, Myrtille Picaud est actuellement en thèse de sociologie à l'École des hautes études en sciences sociales (EHESS) et rattachée au Centre Européen de Sociologie et de Science Politique (CESSP). Elle travaille sur les salles de musique et leurs programmateurs et programmatrices à Paris et à Berlin et sur les intermédiaires culturels. Ses enquêtes précédentes portaient sur les avant-gardes, la marginalité, les scènes virtuelles et les pratiques sexuelles.

\section{MATHILDE PROVANSAL}

Ancienne élève de l'ENS de Lyon, agrégée de sciences économiques et sociales, Mathilde Provansal est en doctorat de sociologie à l'Université Paris 1 Panthéon-Sorbonne et rattachée au laboratoire de l'IDHES (Institutions et Dynamiques Historiques de l'Économie et de la Société). Elle travaille sur la féminisation du monde de l'art contemporain à travers une analyse des trajectoires professionnelles, conjugales et familiales des ancien-ne-s étudian-e-s d'une école des beaux-arts française. 